\title{
Sporadic Cryptosporidium infection in Nigerian children: risk factors with species identification
}

\author{
S. F. MOLLOY ${ }^{1 *}$, C. J. TANNER ${ }^{1}$, P. KIRWAN¹, S. O. ASAOLU ${ }^{2}$, H. V. SMITH ${ }^{3}$, \\ R. A. B. NICHOLS ${ }^{3}$, L. CONNELLY ${ }^{3}$ AND C. V. HOLLAND ${ }^{1}$ \\ ${ }^{1}$ Zoology Department, School of Natural Sciences, Trinity College Dublin, Dublin, Ireland \\ ${ }^{2}$ Scottish Parasite Diagnostic Laboratory, Stobhill NHS Trust, Glasgow, Scotland \\ ${ }^{3}$ Zoology Department, Obafemi Awolowo University, Ile-Ife, Osun State, Nigeria
}

(Accepted 4 August 2010; first published online 27 August 2010)

\section{SUMMARY}

A cross-sectional study was conducted to investigate risk factors for sporadic Cryptosporidium infection in a paediatric population in Nigeria. Of 692 children, $134(19 \cdot 4 \%)$ were infected with Cryptosporidium oocysts. Cryptosporidium spp. were identified in 49 positive samples using PCR-restriction fragment length polymorphism and direct sequencing of the glycoprotein 60 (GP60) gene. Generalized linear mixed-effects models were used to identify risk factors for all Cryptosporidium infections, as well as for C. hominis and C. parvum both together and separately. Risk factors identified for all Cryptosporidium infections included malaria infection and a lack of Ascaris infection. For C. hominis infections, stunting and younger age were highlighted as risk factors, while stunting and malaria infection were identified as risk factors for C. parvum infection.

Key words: Cryptosporidium, epidemiology, infectious disease epidemiology, molecular epidemiology, parasitic disease epidemiology and control.

\section{INTRODUCTION}

Cryptosporidium is a ubiquitous protozoan parasite, infecting many vertebrate hosts, including humans, and is the causative agent of the diarrhoeal disease cryptosporidiosis. Infection may be asymptomatic, mild and self-limiting in immunocompetent patients or severe and prolonged in the immunocompromised [1]. Effective chemotherapeutic treatment for cryptosporidiosis has yet to be developed, so identifying risk factors for Cryptosporidium infection plays an essential role in effectively reducing exposure to infectious oocysts. It is generally accepted that Cryptosporidium

* Author for correspondence: Dr S. F. Molloy, Zoology Department, School of Natural Sciences, Trinity College Dublin, Dublin 2, Ireland.

(Email: molloysi@tcd.ie) can be transmitted by a number of routes such as person-to-person contact, animal-to-person contact, consumption of contaminated drinking/recreational water and food, and contact with contaminated environmental surfaces [2]. The importance and significance of each transmission route for infection is likely to differ between regions and populations.

A number of studies have been carried out in both the developed and the developing world to determine possible risk factors for Cryptosporidium infection. However, to date the majority of studies have focused on cryptosporidiosis outbreaks in developed countries [3-5], and this emphasis has lead to a paucity of data relating to risk factors for sporadic infection in general and in particular, within developing regions. Because risk factors are likely to vary between the developed and developing world, we urgently require 
risk-factor analysis from tropical environments in order to better understand the transmission dynamics of Cryptosporidium under varying environmental and societal conditions.

Of the few studies carried out in developing countries, risk factors include younger age ( $<2$ years) [6,7], children that are not breastfed $[6,8]$, contact with pets $[9,10]$, living in overcrowded conditions $[9,11]$, low birth weight [11], gender [12], and malnourishment [8]. The majority of these studies were based in Latin America with few conducted in Sub-Saharan Africa. To date, no study has attempted to determine risk factors for infection in Nigeria, the most populous African country.

Furthermore, most studies conducted in developing regions have not used molecular techniques to identify Cryptosporidium spp. and assess risk factors separately for the different species. Recent evidence suggests that risk factors for C. parvum and C. hominis infection differ [13,14], and so determining the species present for risk-factor analysis is essential. Hunter et al. [13] carried out a case-control study on sporadic cryptosporidiosis in patients in England and Wales. A number of factors were found to be positively associated with risk; however, when risk factors were investigated separately for C. parvum and C. hominis, the main risk factors for $C$. parvum infection (contact with cattle) and $C$. hominis infection (travel abroad and changing diapers) differed. The study indicated that when $C$. parvum and $C$. hominis are grouped, the analysis highlights factors common to both species, but underestimates risk factors that may be associated with one species or the other. Thus, when carrying out such studies, it may be preferable to determine the species of Cryptosporidium causing the infections and to investigate $C$. hominis and $C$. parvum both together and separately for risk-factor analysis.

The current study, therefore, aimed to investigate, for the first time in Nigeria, the key socioeconomic and other risk factors that are important for Cryptosporidium infection in general (all species), as well as for $C$. parvum and C. hominis, within a paediatric population.

\section{MATERIALS AND METHODS}

\section{Study population and sample collection}

This cross-sectional study was carried out in four semi-urban villages, Ipetumodu, Akinlalu, Edunabon and Moro, situated within $15 \mathrm{~km}$ of Ile-Ife town,
Osun state, Nigeria [15]. The area is characterized by a tropical climate, with the rainy season extending from April to October (with a dip in precipitation during August) and the dry season from November to March. Annual rainfall in the region ranges from 1000 to $4000 \mathrm{~mm}$ [16]. The average maximum daily temperature is $31{ }^{\circ} \mathrm{C}$ in January and $20^{\circ} \mathrm{C}$ in June, and the vegetation is predominately rainforest [16].

Houses in the villages are predominantly built of concrete walls and floors and roofed with galvanized iron sheets. There is no organized sewage disposal system, and refuse and human faeces are dumped in the bush behind the houses or burned [16]. The main source of water is from shared community taps and/or wells located in each village [15]. Animals roam freely throughout the villages. Dogs, goats and chickens are the most commonly observed while cats and ducks are also present, but in lower numbers. Cows are generally kept on farms outside the villages, and therefore, rarely come in contact with children.

The current study was part of a parallel project that ran from May 2006 to August 2007 to assess the prevalence of malaria and Ascaris infection [15]. Malaria infection was determined using a Parascreen rapid diagnostic test (RDTs; Zephyr Biomedicals, India) according to the manufacturer's instructions, and stool samples were examined for Ascaris infection by means of the modified formol-ether concentration technique as previously described [15]. Children were enrolled into the parallel project during May and September 2006, following informed consent. Temporary clinics were set up in the town hall of each village, and mothers from the surrounding area were asked to bring their children for screening of malaria and intestinal worms at four time-points over a 1-year period. The current project was conducted during the last phase of the parallel study, in August 2007. A single stool sample was collected from each child attending the clinics at this time.

\section{Interviews and nutritional status}

In August 2007, detailed questionnaires were administered, by trained field workers, to the mother of each child enrolled in the study. Interviews were conducted in Yoruba and the answers translated into English. Information on age and gender of the child, socioeconomic status of the family, hygiene practices, sources and treatment of drinking water and food, and animal exposure was collected. Furthermore, the nutritional status of each child was assessed by measuring 
anthropometrics. The weight of each child was measured using an electronic balance and height was measured using a stadiometer. Using height and weight data, anthropometric indices ( $z$ scores) for weightfor-age (underweight), height-for-age (stunting) and weight-for-height (wasting) were calculated [17].

A total of 692 children attended the August clinic, providing completed questionnaires and submitting stool samples. Stool samples were collected within 2 days following the administration of the questionnaires. An infected individual was described as a child with Cryptosporidium oocysts in the stool at that time. Presence or absence of diarrhoea was not taken into consideration as the aim of the current study was to determine risk factors for Cryptosporidium infection rather than cryptosporidiosis.

Ethical clearance was granted by the Ethics and Research Committee, Obafemi Awolowo University Teaching Hospital Complex, Ile-Ife, Nigeria.

\section{Stool analysis}

Stool consistency was evaluated by visual examination of the stool on the day of sample collection, and was classified as formed, unformed or liquid. Subsequently, samples were transported to Trinity College Dublin, unfixed, and refrigerated at $4{ }^{\circ} \mathrm{C}$. Fluorescent microscopy, following modified formol-ether concentration and auramine phenol staining, was undertaken to determine the presence of Cryptosporidium oocysts in the stool using previously described methods [18]. Samples were processed within 8 weeks of sample collection. A $\chi^{2}$ analysis was used to test for an association between infection status and stool consistency. The intensity of infection was recorded for each positive sample. Intensity was determined as follows: $0=$ no oocysts detected in the sample; $1+=$ $1-10$ oocysts per field of view $; 2+=10-50$ oocysts per field of view, and $3+=>50$ oocysts per field of view.

\section{Genotyping}

All 134 positive samples were concentrated by waterether concentration and DNA was extracted using 15 cycles of freeze-thawing [19]. Nested PCR-RFLP or PCR sequencing at two SSU rRNA gene loci was performed as previously described [18]. Genotyping was performed in the Scottish Parasite Diagnostic Laboratory, Stobhill NHS Trust, Glasgow. Samples were stored unfixed at $4{ }^{\circ} \mathrm{C}$ and were processed within 18 months of sample collection.

\section{Statistical analysis}

The 692 questionnaires were validated using consistency checks, and records with errors were corrected from the original questionnaire. All statistical analyses were performed using the lme4 package [20] in $\mathrm{R}$ version 2.9.0 [21].

A number of scoring indices were constructed to describe the population. These included a socioeconomic index, a hygiene index and an animal contact frequency index. Variables relating to each index were categorized, ranked and scored (Table 1). The scores allocated to the variables for each index were added, with a low index number indicating low socioeconomic status, low hygiene and/or contact with few animals. The child and adult hygiene indices were highly correlated (correlation coefficient $=0 \cdot 377$, $P<0.001$ ), therefore an average of the two indices was used to describe the condition of parent and child. Metrics describing each child's nutritional status were also recorded. The weight-for-height (wasting) metric was highly correlated to both height-for-age (stunting) (correlation coefficient $=0.367, P<0.001$ ) and weight-for-age (underweight) (correlation coefficient $=$ $0 \cdot 271, P<0 \cdot 001$ ), but weight-for-age (underweight) and height-for-age (stunting) were not correlated (correlation coefficient $=0 \cdot 011, P=0 \cdot 777$ ). Therefore, weight-for-height was excluded from the analysis.

The socioeconomic index was significantly correlated with the level of maternal education (correlation coefficient $=0 \cdot 450, P<0 \cdot 001$ ), hygiene index (correlation coefficient $=0 \cdot 128, P<0 \cdot 001)$ and animal index (correlation coefficient $=-0 \cdot 147, P<0 \cdot 001$ ). Therefore, models in which the full model included either socioeconomic index, level of maternal education, hygiene index, or animal index were each analysed independently, and the best-fitting model based on Akaike's Information Criterion (AIC) was retained [22]. In such cases, we therefore cannot rule out correlated factors. Thus, although we present the best model fit given the data, there are correlations between factors that should not be disregarded in future studies.

To determine which factors contribute to the presence of sporadic Cryptosporidium infection in children, we used a series of generalized linear mixedeffects models with a binomial distribution. These models allow for the investigation of hierarchical or nested data in which the explanatory variables are not completely independent and the response variable comes from a non-normal distribution [20, 23]. As 
Table 1. Construction of socioeconomic, hygiene and animal indices

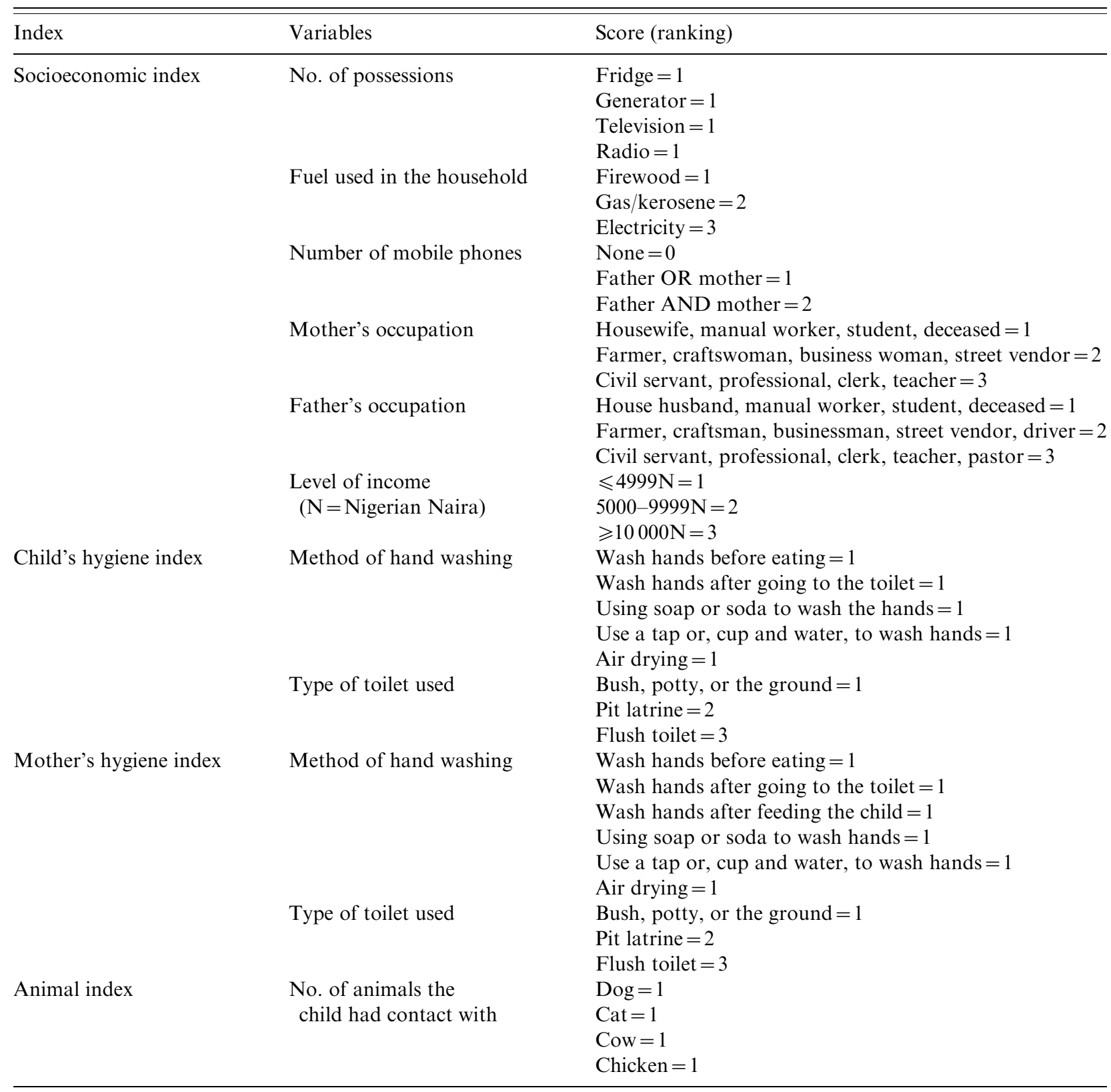

such, they offer a methodological advance in hierarchical analysis analogous to the conceptual framework suggested by Victora et al. [24]. Fixed effects in the initial full model for all Cryptosporidium infections included the child's age, socioeconomic index score, height-for-age (stunting), weight-for-age (underweight), gender, animal contact index, household crowding, average hygiene index, malaria infection, Ascaris infection, boiling of drinking water, and diarrhoea symptoms of household members. We also included all two- and three-way interactions between explanatory variables in the initial full model to investigate the potential for interactions among these variables, which can mask their main effects [23]. A child's village was treated as a random effect. Model selection proceeded by stepwise selection basing decisions to keep/drop explanatory variables on parsimony and the resulting fit of each model to the data using AIC values (i.e. explanatory variables were retained in the model if their removal significantly reduced the model fit to the data [22]).

Because infected individuals could have many different types of Cryptosporidium infections, each with associated risk factors, three additional analyses were 
Table 2. Species of Cryptosporidium isolated from a Nigerian paediatric population

\begin{tabular}{lc}
\hline \hline Cryptosporidium species & No. $(\%)$ \\
\hline C. hominis & $19(37 \cdot 3)$ \\
C. parvum & $18(35 \cdot 3)$ \\
C. parvum/C. hominis & $4(7 \cdot 8)$ \\
C. meleagridis & $4(7 \cdot 8)$ \\
Cryptosporidium rabbit genotype & $2(3 \cdot 9)$ \\
Cryptosporidium cervine genotype & $1(2 \cdot 0)$ \\
C. canis & $1(2 \cdot 0)$ \\
Total & $49(100)$ \\
\hline \hline
\end{tabular}

performed to determine whether risk factors differed for C. hominis and C. parvum relative to all Cryptosporidium infections combined. Only 49/134 samples amplified using PCR-RFLP, therefore, species data for only 49 samples were available for subsequent risk-factor analyses. The first analysis was for samples positive for either C. hominis or C. parvum; the second was for $C$. hominis only, and the third was for C. parvum only. These analyses were performed as described above for the full model, except that interactions were not included in the versions with only C. hominis or C. parvum to be conservative with lowsample sizes.

\section{RESULTS}

A total of 692 children submitted a sample and completed a questionnaire in August 2007. Almost equal numbers of males and females attended, 335 males $(48.4 \%)$ and $357(51.6 \%)$ females. Children were aged between $19 \cdot 5$ and 72 months, with a median of 43.7 months. A total of $148(21 \cdot 4 \%)$ children were from the village of Akinlalu, 298 (43.1\%) from Ipetumodu, $84(12 \cdot 1 \%)$ from Moro and $162(23 \cdot 4 \%)$ from Edunabon.

Of the 692 children, Cryptosporidium oocysts were detected in 134 samples, $19 \cdot 4 \%$ (95\% CI 16.42-23). The majority of infections had a low intensity $(88.8 \%$, $1+$ ), while $2 \cdot 3 \%$ were medium intensity $(2+)$ infections and $8.2 \%$ were of high intensity $(3+)$. Although diarrhoeal status was not determined conclusively, stool consistency was noted. There was a significant association between stool consistency and infection status $\left(\chi^{2}=6 \cdot 04\right.$, D.F. $\left.=2, P=0 \cdot 049\right)$. A lower percentage of infected individuals passed formed stools ( $26 \cdot 1 \%)$ compared to uninfected individuals $(30 \cdot 1 \%)$. A low number of watery samples were collected
Table 3. C. parvum, C. hominis, malaria and Ascaris infections distributed by age, gender and village

\begin{tabular}{|c|c|c|c|c|c|}
\hline Demographic & $N_{\text {study }}$ & $\begin{array}{l}C . \\
\text { hominis }\end{array}$ & $\begin{array}{l}C . \\
\text { parvum }\end{array}$ & Malaria & Ascaris \\
\hline \multicolumn{6}{|l|}{ Age (yr) } \\
\hline$<2$ & 40 & 1 & 2 & 26 & 11 \\
\hline $2-4$ & 379 & 14 & 9 & 264 & 109 \\
\hline$>4$ & 273 & 4 & 7 & 192 & 70 \\
\hline \multicolumn{6}{|l|}{ Gender } \\
\hline Male & 335 & 11 & 9 & 229 & 96 \\
\hline Female & 357 & 8 & 9 & 253 & 94 \\
\hline \multicolumn{6}{|l|}{ Village } \\
\hline Akinlalu & 148 & 2 & 4 & 110 & 49 \\
\hline Ipetumodu & 298 & 7 & 9 & 185 & 83 \\
\hline Edunabon & 162 & 7 & 4 & 122 & 38 \\
\hline Moro & 84 & 3 & 1 & 65 & 20 \\
\hline
\end{tabular}

( $n=20)$, thus reducing our ability to discern differences based on consistency.

All 134 positive stool samples were analysed using two nested PCR-RFLP and/or direct sequencing of PCR products. Of these, 49 samples produced sufficient product for RFLP determination of species. The reasons for the low number of samples amplifying were not determined conclusively, but may have been due to a prolonged period of storage prior to analysis. Although subtyping analysis was performed, the sample size was too low to include in the riskfactor analysis. Where required, DNA sequencing was used to confirm Cryptosporidium spp./genotypes. C. hominis accounted for $37 \cdot 3 \%$ (19) samples, $35 \cdot 3 \%$ (18) C. parvum samples were isolated and there were mixtures of the two species in $7 \cdot 8 \%$ (4) of samples. Other species isolated included, C. meleagridis $(4,7 \cdot 8 \%)$, Cryptosporidium rabbit genotype $(2,3 \cdot 9 \%)$, Cryptosporidium cervine genotype $(1,2.0 \%)$ and C. canis $(1,2.0 \%)$ (Table 2$)$. The distribution of C. hominis and C. parvum by age, gender and village is presented in Table 3.

The final model for all Cryptosporidium infections ( $n=692,134$ positive) included the explanatory variables listed in Table 4. Children were more at risk of being infected with Cryptosporidium if they were infected with malaria, but less at risk if they were infected with Ascaris. Furthermore, interactions between explanatory variables served to mask some of the main effects themselves, suggesting that the effects of these factors are not independent of the presence of particular values of other factors in the analysis. The interaction between household crowding and a household member with diarrhoea indicates that when 
Table 4. Results of generalized linear mixed-effects models for (a) all Cryptosporidium infections $($ AIC $=624 \cdot 5)$, (b) C. hominis or C. parvum infections $(A I C=277 \cdot 1)$, (c) C. hominis infections only $(A I C=174 \cdot 5)$, and (d) C. parvum infections only $(A I C=163 \cdot 4)$

\begin{tabular}{|c|c|c|c|c|c|}
\hline Infected group & Factor & Estimate & S.E. & $z$ & $P$ \\
\hline $\begin{array}{l}\text { (a) All Cryptosporidium infections } \\
(n=692,134 \text { infected })\end{array}$ & $\begin{array}{l}\text { Socioeconomic index } \\
\text { Gender } \\
\text { Family member with diarrhoea } \\
\text { Household crowding } \\
\text { Malaria infection } \\
\text { Ascaris infection } \\
\text { Diarrhoea symptoms x crowding } \\
\text { Gender x socioeconomic index }\end{array}$ & $\begin{array}{r}0 \cdot 062 \\
2 \cdot 334 \\
-2 \cdot 817 \\
-0 \cdot 017 \\
0 \cdot 503 \\
-0 \cdot 770 \\
5 \cdot 482 \\
-0 \cdot 215\end{array}$ & $\begin{array}{l}0 \cdot 071 \\
1 \cdot 251 \\
1 \cdot 621 \\
0 \cdot 310 \\
0 \cdot 245 \\
0 \cdot 258 \\
2 \cdot 562 \\
0 \cdot 105\end{array}$ & $\begin{array}{r}0 \cdot 872 \\
1 \cdot 866 \\
-1 \cdot 738 \\
-0 \cdot 056 \\
2 \cdot 053 \\
-2 \cdot 987 \\
2 \cdot 140 \\
-2 \cdot 049\end{array}$ & $\begin{array}{l}0 \cdot 383 \\
0 \cdot 062 \\
0 \cdot 082 \\
0 \cdot 956 \\
0 \cdot 040 \\
0 \cdot 003 \\
0 \cdot 032 \\
0 \cdot 040\end{array}$ \\
\hline $\begin{array}{l}\text { (b) C. hominis and C.parvum only } \\
(n=692,37 \text { infected })\end{array}$ & $\begin{array}{l}\text { Age } \\
\text { Mother's education } \\
\text { Stunting } \\
\text { Malaria infection }\end{array}$ & $\begin{array}{r}-0.032 \\
-0.469 \\
0.407 \\
0.997\end{array}$ & $\begin{array}{l}0 \cdot 013 \\
0 \cdot 237 \\
0 \cdot 145 \\
0 \cdot 493\end{array}$ & $\begin{array}{r}-2 \cdot 408 \\
-1 \cdot 976 \\
2 \cdot 805 \\
2 \cdot 021\end{array}$ & $\begin{array}{l}0 \cdot 016 \\
0 \cdot 048 \\
0 \cdot 005 \\
0 \cdot 043\end{array}$ \\
\hline $\begin{array}{l}\text { (c) C. hominis only } \\
(n=692,19 \text { infected })\end{array}$ & $\begin{array}{l}\text { Age } \\
\text { Stunting }\end{array}$ & $\begin{array}{r}-0 \cdot 036 \\
0 \cdot 367\end{array}$ & $\begin{array}{l}0 \cdot 020 \\
0 \cdot 190\end{array}$ & $\begin{array}{r}-1.942 \\
1.929\end{array}$ & $\begin{array}{l}0 \cdot 052 \\
0 \cdot 054\end{array}$ \\
\hline $\begin{array}{l}\text { (d) C. parvum only } \\
(n=692,18 \text { infected })\end{array}$ & $\begin{array}{l}\text { Stunting } \\
\text { Malaria infection }\end{array}$ & $\begin{array}{l}0 \cdot 443 \\
2 \cdot 000\end{array}$ & $\begin{array}{l}0 \cdot 212 \\
1 \cdot 033\end{array}$ & $\begin{array}{l}2 \cdot 089 \\
1.925\end{array}$ & $\begin{array}{l}0 \cdot 037 \\
0 \cdot 054\end{array}$ \\
\hline
\end{tabular}

AIC, Akaike's Information Criterion.

(a)

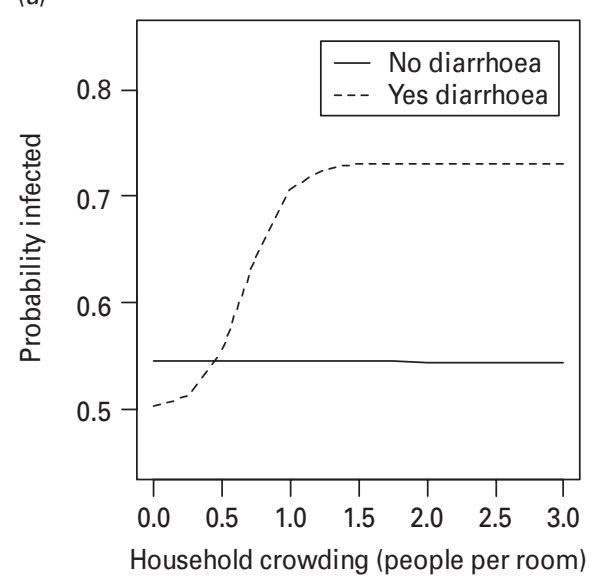

(b)

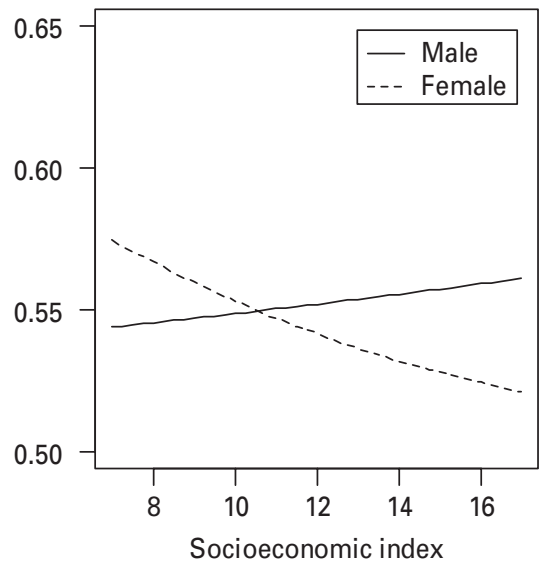

Fig. 1. Interaction plots of how the probability of Cryptosporidium infection changes with either (a) household crowding, or (b) socioeconomic index. (a) A family member exhibiting symptoms of diarrhoea increases the probability of a child being infected only in crowded living conditions. (b) Males and females are affected differently by socioeconomic status. As socioeconomic status increases, the probability of a male being infected increases, while the probability of a female being infected decreases.

household crowding is low, the presence or absence of a family member with diarrhoea has no effect on infection risk. However, as household density increases children living with a family member that has diarrhoea are more at risk of infection than are children with no family members with diarrhoea (Fig. 1a). Therefore, a family member with diarrhoea represents a context-dependent risk factor that is only significant under certain household conditions. Similarly, the interaction between socioeconomic status and a child's gender indicates that, as standard of living increases, a male child's risk of infection increases slightly, while the risk for a female child decreases significantly (Fig. 1b). Again, socioeconomic status represents a context-dependent factor that affects male and female children differently in this study.

When including infections with either $C$. hominis or C. parvum ( $n=692,37$ positive; Table 4$)$, risk 
decreased with an increase in a child's age, as well as with an increase in mother's education, while infection risk increased with severity of stunting and malaria infection. For $C$. hominis infections only (19 positive; Table 4), risk decreased as a child's age increased and as the mother's education increased. Risk of infection increased with severity of stunting. For C. parvum infections only (18 positive; Table 4), risk increased with severity of stunting and malaria infection.

\section{DISCUSSION}

Prevalence of Cryptosporidium in this study was relatively high (19.4\%) compared to similar studies from tropical countries. According to O'Donoghue [1], the prevalence of Cryptosporidium infection in developing countries ranges from $0.1 \%$ to $31.5 \%$ (mean of 48 surveys, $7.9 \%$ ) and the reported prevalence of Cryptosporidium infection from Kenya, Bangladesh, southern India and Brazil in children, aged $<5$ years, with diarrhoea, was $4 \%, 2.6 \%, 7.6 \%$, and $31.2 \%$, respectively $[7,11,25,26]$.

Our study identified a number of risk factors for sporadic Cryptosporidium infection that varied by species. Risk factors identified included infection with malaria, stunting, younger age and low levels of maternal education or socioeconomic status. In addition, children who were not infected with Ascaris, children living in a crowded household and with a member suffering from diarrhoea, and female children in families with low socioeconomic status were at a greater risk of acquiring sporadic Cryptosporidium infection, but these factors applied only to all Cryptosporidium infections.

Previous research has shown that patients can be co-infected with Cryptosporidium and parasites such as Blastocystis hominis, Giardia spp., Entamoeba histolytica, Ascaris lumbricoides, Trichuris trichiura, Strongyloides stercoralis and Dicrocoelium dendriticum [27-29]. However, the majority of these studies simply detected the presence of these species through the analysis of stool samples, with few determining the physiological and immunological significance of such co-occurrences. In the current study, malaria infection was highlighted as a risk factor for all Cryptosporidium infections, for $C$. hominis and $C$. parvum together and for $C$. parvum alone. A common host susceptibility to protozoan infections might explain the increased risk of Cryptosporidium infection in children who had malaria. Alternatively, children may be at risk for both malaria and Cryptosporidium infections due to common environmental factors. In relation to Ascaris infection, children infected with Ascaris were less likely to also be infected with Cryptosporidium and this was observed for all Cryptosporidium infections only. This association between Cryptosporidium and Ascaris has not been previously identified. Thus, due to the paucity of data, the association between Cryptosporidium and malaria and Cryptosporidium and Ascaris merits further investigation.

Stunting (height-for-age) was associated with C. parvum and C. hominis infections combined and with $C$. parvum and $C$. hominis separately in our analysis. However, the direction of causation could not be identified, and so we cannot determine whether stunting is caused by the infection or whether children are predisposed to infection when stunted. Previous studies from India and Peru have also identified a similar relationship between Cryptosporidium infection and stunting $[8,30]$.

Younger age was also identified as a risk factor for C. parvum and C. hominis infections combined and for $C$. hominis only, suggesting that younger children are at higher risk of infection if $C$. hominis is circulating in the population rather than $C$. parvum. This is in contrast to findings in southern India that found no significant differences in age between $C$. hominis-infected children and those infected with other species [25], therefore further investigation is required.

An interaction between socioeconomic status and gender was observed for all Cryptosporidium infections such that girls were less likely to become infected if they belonged to a family with a high socioeconomic status. In the current study, as socioeconomic status and levels of maternal education were highly correlated, it is possible that educated mothers pass on their knowledge to their daughters more so than to their sons. Low levels of maternal education were highlighted as a risk factor for C. parvum and C. hominis infections together. However, few studies have identified parental education and/or socioeconomic status as a risk factor for Cryptosporidium infection. In a number of studies, it has been suggested that a mother's education is an important protective factor for children, against infection with a range of intestinal parasites [31, 32], indicating that parents with a higher level of education may exhibit behaviours (e.g. hygiene awareness) that would reduce exposure to various infections in children. 
An interaction between the presence of a member in the household with diarrhoea and household crowding was observed for all Cryptosporidium infections. Results indicated that the presence of people in the household with diarrhoea becomes important in crowded households (possibly due to increased person-to-person contact) and leads to an increased risk of infection. Direct person-to-person transmission has been observed previously in nurseries, and within both hospitals and families [33-35].

The current study is the first to identify risk factors for sporadic Cryptosporidium infection in Nigeria and the first in Africa to consider risk factors separately for $C$. hominis and C. parvum species. The role of parasite genetics in risk-factor analysis is not entirely clear, but recent data have indicated that clinical manifestations for Cryptosporidium vary between species and subtypes [36-38], and there is evidence to suggest that distinct sources, and therefore transmission routes, exist for C. hominis and C. parvum due to variations observed in host ranges (with $C$. hominis limited primarily to human infections and $C$. parvum linked to both zoonotic and anthroponotic infection). This indicates that sufficient variation exists between species and subtypes such that risk factors for infection may also differ. To date, only five studies, two from the developed world (UK) and three from developing regions (Peru and India) have assessed risk factors separately by species [13, 14, 25, 37, 38].

Of the three large-scale studies conducted in developing countries, two studies failed to identify any risk factors for infection for a range of Cryptosporidium spp. and subtypes even though a wide range of behavioural and social variables were analysed $[25,37,38]$. In the third study, based in Peru, only one risk factor was identified. HIV-infected individuals who had been in contact with children aged $<5$ years were more likely to be infected with $C$. hominis subtype family Ie [38].

Our study provides a mixed picture with one common risk factor, stunting (height-for-age), for both species whereas infection with malaria was only associated with C. parvum infection, and younger age only with $C$. hominis infection. These differences may reflect a common host susceptibility to parasitic infection for C. parvum-infected children, although due to the low sample size this cannot be determined conclusively. Our results provide novel and important data regarding risk factors for sporadic Cryptosporidium infection in an African context; however, information to enable implementation of specific control measures to reduce transmission of infectious oocysts is limited. Future studies should be conducted, in various tropical regions, which include both larger sample sizes for C. hominis and C. parvum and subtyping data. This will enable researchers to elucidate more clearly the role of parasite genetics in the identification of risk factors for Cryptosporidium infection.

\section{ACKNOWLEDGEMENTS}

We thank all the mothers and children that took part in this study and all Obas, field workers, doctors and nurses for their excellent work. Síle Molloy was the recipient of a Ph.D. scholarship from the Irish Research Council for Science, Engineering and Technology. The fieldwork for this project was funded by a Health Research Board grant (GHRA/ 2006/7). Colby Tanner was funded by the Irish Research Council for Science, Engineering and Technology INSPIRE fellowship.

\section{DECLARATION OF INTEREST}

None.

\section{REFERENCES}

1. O'Donoghue PJ. Cryptosporidium and cryptosporidiosis in man and animals. International Journal for Parasitology 1995; 25: 139-195.

2. Kosek M, et al. Cryptosporidiosis: an update. Lancet Infectious Diseases 2001; 1: 262-269.

3. Osewe $\mathbf{P}$, et al. Cryptosporidiosis in Wisconsin: a case-control study of post-outbreak transmission. Epidemiology and Infection 1996; 117: 297-304.

4. Quiroz ES, et al. An outbreak of cryptosporidiosis linked to a foodhandler. Journal of Infectious Diseases 2000; 181: 695-700.

5. Stafford R, et al. A community outbreak of Cryptosporidium infection associated with a swimming pool complex. Communicable Disease Report 2000; 24: 236-239.

6. Abdel-Messih IA, et al. Diarrhea associated with Cryptosporidium parvum among young children of the Nile River Delta in Egypt. Journal of Tropical Pediatrics 2005; 51: 154-159.

7. Khan WA, et al. Cryptosporidiosis among Bangladeshi children with diarrhea: a prospective, matched, casecontrol study of clinical features, epidemiology and systemic antibody responses. American Journal of Tropical Medicine and Hygiene 2004; 71: 412-419.

8. Bhattacharya MK, et al. Cryptosporidium infection in children in urban Bangladesh. Journal of Tropical Pediatrics 1997; 43: 282-286. 
9. Katsumata $\mathbf{T}$, et al. Cryptosporidiosis in Indonesia: a hospital-based study and a community-based survey. American Journal of Tropical Medicine and Hygiene 1998; 59: 628-632.

10. Xiao L, et al. Possible transmission of Cryptosporidium canis among children and a dog in a household. Journal of Clinical Microbiology 2007; 45: 2014-2016.

11. Newman RD, et al. Longitudinal study of Cryptosporidium infection in children in northeastern Brazil. Journal of Infectious Diseases 1999; 180: 167-175.

12. Pereira MD, et al. Intra-familial and extra-familial risk factors associated with Cryptosporidium parvum infection among children hospitalized for diarrhea in Goiania, Goias, Brazil. American Journal of Tropical Medicine and Hygiene 2002; 66: 787-793.

13. Hunter PR, et al. Sporadic cryptosporidiosis casecontrol study with genotyping. Emerging Infectious Diseases 2004; 10: 1241-1249.

14. Lake IR, et al. Case-control study of environmental and social factors influencing cryptosporidiosis. European Journal of Epidemiology 2007; 22: 805-811.

15. Kirwan $\mathbf{P}$, et al. Patterns of soil-transmitted helminth infection and impact of four-monthly albendazole treatments in preschool children from semi-urban communities in Nigeria: a double-blind placebocontrolled randomised trial. BMC Infectious Disease 2009; 9: 20.

16. Asaolu SO, Holland CV, Crompton DW. Community control of Ascaris lumbricoides in rural Oyo State, Nigeria: mass, targeted and selective treatment with levamisole. Parasitology 1991; 103: 291-298.

17. Cogill B. Anthropmetric Indicators Measurement Guide. Food and Nutrition Technical Assistance (FANTA) (http://www.fantaproject.org). Washington, DC: Academy for Educational Development Project, 2003, pp. 92.

18. Molloy SF, et al. Identification of a high diversity of Cryptosporidium species, genotypes and subtypes in a Nigerian pediatric population. American Journal of Tropical Medicine and Hygiene 2009; 82: 608-613.

19. Nichols RAB, Moore JE, Smith HV. A rapid method for extracting oocyst DNA from Cryptosporidium-positive human faeces for outbreak investigations. Journal of Microbiological Methods 2006; 65: 512-524.

20. Bates D, Maechler M. lme4: linear mixed-effects models using S4 classes. R package version 0.999375-32 (http:// CRAN.R-project.org/package =lme4), 2009.

21. R Development Core Team. $R: A$ Language and Environment for Statistical Computing. Vienna, Austria (http://www.R-project.org), 2009.

22. Burnham KP, Anderson DR. Model Selection and Multimodel Inference: A Practical Information-Theoretic Approach. New York: Springer, 2002, pp. 448.

23. Crawley MJ. The $R$ Book. New York: Wiley \& Sons, 2007, pp. 942.
24. Victora CG, et al. The role of conceptual frameworks in epidemiological analysis: a hierarchical approach. International Journal of Epidemiology 1997; 26: 224 227.

25. Ajjampur SS, et al. Molecular and spatial epidemiology of cryptosporidiosis in children in a semiurban community in South India. Journal of Clinical Microbiology 2007; 45: 915-920.

26. Gatei W, et al. Cryptosporidiosis: Prevalence, genotype analysis, and symptoms associated with infections in children in Kenya. American Journal of Tropical Medicine and Hygiene 2006; 75: 78-82.

27. Kurniawan A, et al. Intestinal parasitic infections in HIV/AIDS patients presenting with diarrhoea in Jakarta, Indonesia. Transactions of the Royal Society of Tropical Medicine and Hygiene 2009; 103: 892-898.

28. Mukherjee AK, et al. Hospital-based surveillance of enteric parasites in Kolkata. BMC Research Notes 2009; 2: 110 .

29. Nwabuisi C. Childhood cryptosporidiosis and intestinal parasitosis in association with diarrhoea in Kwara State, Nigeria. West African Journal of Medicine 2001; 20: $165-168$.

30. Checkley W, et al. Effects of Cryptosporidium parvum infection in Peruvian children: growth faltering and subsequent catch-up growth. American Journal of Epidemiology 1998; 148: 497-506.

31. Quihui L, et al. Role of the employment status and education of mothers in the prevalence of intestinal parasitic infections in Mexican rural schoolchildren. BMC Public Health 2006; 6: 225.

32. Nematiana $\mathbf{J}$, et al. Prevalence of intestinal parasitic infections and their relation with socio-economic factors and hygienic habits in Tehran primary school students. Acta Tropica 2004; 92 : 179-186.

33. Fayer R, Morgan U, Upton SJ. Epidemiology of Cryptosporidium: transmission, detection and identification. International Journal for Parasitology 2000; 30 : 1305-1322.

34. Rahman M, et al. Cryptosporidiosis : a cause of diarrhea in Bangladesh. American Journal of Tropical Medicine and Hygiene $1990 ;$ 42: 127-130.

35. Navarrete S, et al. An outbreak of Cryptosporidium diarrhea in a pediatric hospital. Pediatric Infectious Disease Journal 1991; 10: 248-520.

36. Bushen OY, et al. Heavy cryptosporidial infections in children in northeast Brazil: comparison of Cryptosporidium hominis and Cryptosporidium parvum. Transactions of the Royal Society of Tropical Medicine and Hygiene 2007; 101: 378-384.

37. Cama VA, et al. Cryptosporidium species and subtypes and clinical manifestations in children, Peru. Emerging Infectious Diseases 2008; 14: 1567-1574.

38. Cama VA, et al. Differences in clinical manifestations among Cryptosporidium species and subtypes in HIVinfected persons. Journal of Infectious Diseases 2007; 196: 684-691. 